\title{
Reliable Computation of Homogeneous Azeotropes
}

\author{
R. W. Maier, M. A. Stadtherr, J. F. Brennecke \\ Midwest Thermodynamics Conference, Notre Dame, IN, May 17-19, 1998
}

\begin{abstract}
The determination of the existence and composition of azeotropes is important both from theoretical and practical standpoints. An important test of the veracity of thermodynamic models is whether or not known azeotropes are predicted, and whether or not they are predicted accurately. Model parameters can be fine tuned by comparing the model predictions can be used as starting points for experimental searches for actual azeotropes. These azeotropes often present limitations in process design which must be known, and their determination strictly from experiment alone can be expensive.

There are two main difficulties in solving the problem. The first is the fact that the equations derived from most thermodynamic models are highly nonlinear, which may make finding any azeotrope a nontrivial exercise. In addition, there is the question of whether or not all of the azeotropes have been found, or of being certain that there are no azeotropes if none have been found. Because of these difficulties there has been much recent interest in the reliable computation of azeotropes, focused primarily on the prediction of homogeneous azeotropes. For example, Fidkowski et al. (1993) have present a homotopy continuation method for the calculation of homogeneous azeotropes. The primary drawback of this technique is that it can not guarantee that all azeotropes have been found. More recently, Harding et al. (1997) have reported a global optimization procedure based on a branch and bound approach using convex underestimation functions that are continuously refined as the range where azeotropes are possible is narrowed. This technique does provide a guarantee that all azeotropes have been found. Harding et al. (1997) have developed appropriate convex underestimating functions for several specific thermodynamic models.

We describe here a new approach for reliably finding all homogeneous azeotropes of multicomponent mixtures. The technique is based on interval analysis, in particular the use of an interval Newton/generalized bisection algorithm. The method can determine with mathematical certainty all azeotropes for any system. The technique is general purpose and can be applied in connection with any thermodynamic models. No model specific convex underestimating functions need be derived. In this paper, the technique is described in detail, and then tested on several problems. Results of the test problems indicate that the method can efficiently and reliably determine all homogeneous azeotropes for multicomponent mixtures.
\end{abstract}

\section{References}

Fidkowski, Z. T., M. F. Malone and M. F. Doherty, Computing azeotropes in multicomponent mixtures. Comput. Chem. Eng., 17, 1141-1155 (1993).

S. T. Harding, C. D. Maranas, C. M. McDonald and C. A. Floudas, Locating All Homogeneous Azeotropes in Multicomponent Mixtures. Ind. Eng. Chem. Res., 36, 160-178 (1997). 